\title{
The Current Practice of Skin Testing for Antibiotics in Korean Hospitals
}

\author{
So Hee Lee, Heung Woo Park, Sae Hoon Kim, Yoon Seok Chang, Sun Sin Kim, Sang Heon Cho, Kyung Up Min, \\ and You Young Kim
}

Department of Internal Medicine, Seoul National University Hospital, Seoul; Department of Internal Medicine, Seoul National University Bundang Hospital, Seongnam, Korea

Background/Aims: Antibiotic skin testing is a useful procedure for identifying patients with IgE-mediated hypersensitivity to antibiotics. The procedures, however, have not been standardized, and the testing is performed with diverse protocols in Korean hospitals wards. Thus, we examined the current practice of antibiotic skin testing in Korea.

Methods: We sent questionnaires to 12 allergists working in secondary or tertiary referral hospitals and collected them by e-mail or fax. The questionnaire included items such as the types and concentrations of the tested antibiotics, the methods of antibiotic skin testing, and the interpretation of the results.

Results: All hospitals responded to the questionnaire. The antibiotic skin testing protocols were variable, inconsistent, and differed with regard to the type and concentrations of antibiotics, the volume injected, and the interpretation of the results. Moreover, the protocols differed from the commonly recommended procedures in the medical literature.

Conclusions: Standardized guidelines for antibiotic skin testing are needed for the safe and effective use of antibiotics in Korea. (Korean J Intern Med 2010;25:207-212)

Keywords: Anti-bacterial agents; Drug hypersensitivity; Skin tests

\section{INTRODUCTION}

Adverse reactions to antibiotic drugs are common, and up to $25 \%$ of patients who are treated with antimicrobial agents report allergic reactions to antimicrobials [1]. Penicillin is the most frequently implicated agent; approximately $10 \%$ to $20 \%$ of hospitalized patients have a history of penicillin allergy $[1,2]$. As the use of semisynthetic penicillins (amoxicillin and ampicillin) and cephalosporins has increased, these agents are becoming a major cause of allergic responses [3]. Generally, nonbeta-lactam antibiotics such as vancomycin and fluoroquinolones are commonly used in patients with a history of beta-lactam allergy to avoid allergic reactions.
The use of alternative antibiotics is associated with an increased number of infections caused by multidrugresistant bacterial strains, which is associated with higher costs for care, morbidity, and mortality [4-7]. To solve this problem, identifying patients in the clinical setting who are at high risk for immediate IgE-mediated reactions is essential. Antibiotic skin testing (AST) is well-known to be a safe and reliable method for detecting immediate allergic reactions to beta-lactams. Although skin testing is a routine antibiotic test in Korean hospital wards, methods vary from hospital to hospital. The purpose of this study was to examine the current practice of AST in Korea. 
Table 1. Types and concentrations of antibiotics tested in 12 hospitals

\begin{tabular}{|c|c|c|c|c|c|c|c|}
\hline Hospitals & $\begin{array}{l}\text { Penicillin G, } \\
\text { IU } / \mathrm{mL}\end{array}$ & $\begin{array}{c}\text { Semisynthetic } \\
\text { penicillin, } \mathrm{mg} / \mathrm{mL}\end{array}$ & $\begin{array}{c}\text { Cephalosporin, } \\
\mathrm{mg} / \mathrm{mL}\end{array}$ & $\begin{array}{c}\text { Carbapenem, } \\
\mathrm{mg} / \mathrm{mL}\end{array}$ & $\begin{array}{c}\text { Vancomycin, } \\
\mathrm{mg} / \mathrm{mL}\end{array}$ & $\begin{array}{c}\text { Streptomycin, } \\
\mathrm{mg} / \mathrm{mL}\end{array}$ & $\begin{array}{c}\text { Monobactam, } \\
\mathrm{mg} / \mathrm{mL}\end{array}$ \\
\hline$A$ & $1 / 100^{a}$ & $1 / 100^{a}$ & $1 / 100^{a}$ & $1 / 100^{a}$ & ND & $1 / 100^{a}$ & ND \\
\hline B & $1 / 100^{a}$ & $1 / 100^{a}$ & $1 / 100^{a}$ & $1 / 100^{a}$ & $1 / 100^{a}$ & ND & ND \\
\hline C & 500 & 0.3 & 0.3 & 0.3 & ND & 0.1 & ND \\
\hline $\mathrm{D}$ & 800 & 0.3 & 0.3 & ND & ND & ND & ND \\
\hline$E$ & 10,000 & 3 & ND & 1 & ND & ND & 3 \\
\hline $\mathrm{F}$ & 5 & 1 & 1 & ND & ND & ND & ND \\
\hline$G$ & 1,500 & 0.3 & 0.3 & 0.3 & ND & ND & ND \\
\hline $\mathrm{H}$ & ND & ND & 20 & 20 & 20 & ND & ND \\
\hline 1 & No use & 0.1 & 0.1 & ND & 0.1 & ND & ND \\
\hline $\mathrm{J}^{\mathrm{b}}$ & 10,000 & 3 & 4 & 4 & ND & 4 & ND \\
\hline $\mathrm{K}^{\mathrm{b}}$ & 50,000 & 5 & 5 & 5 & 5 & ND & ND \\
\hline $\mathrm{L}^{b}$ & 10,000 & 3 & 4 & 4 & ND & ND & ND \\
\hline
\end{tabular}

ND, not done.

a 1 / 100 dilution regardless of the antibiotic type and dose.

b Secondary hospitals; the others are tertiary hospitals.

\section{METHODS}

We selected 12 hospitals that employed allergy specialists. To represent general practices in Korea, we tried to avoid deviations in grade and location of the hospitals, so three secondary hospitals ( $\mathrm{J}$ to L, Table 1 ) and nine tertiary hospitals (A to I, Table 1) were enrolled. Among them, one secondary hospital (J, Table 1 ) and five tertiary hospitals (C to G, Table 1) were located in Seoul, and two secondary hospitals (K and L, Table 1) and four tertiary hospitals (A, B, H, and I, Table 1) were located in the province. Questionnaires were sent to the allergists, and the responses were collected either by e-mail or fax. The questionnaire included items such as the type and concentration of the antibiotics tested, the testing methods, and the interpretation of the test results. The questions were as follows:

1) Do you routinely conduct AST before using antibiotics in your wards? If so, how many and which antibiotics do you test? 2) Clarify the details of the methods for AST, e.g., testing method (skin prick testing vs. intradermal testing), concentrations and doses of antibiotics tested, test site, and whether a control substance is tested. 3) Clarify the method for AST interpretation, e.g., time lag between testing and interpretation and criteria for positivity.

\section{RESULTS}

The response rate was $100 \%$. In all hospitals, the AST was a routine procedure before starting certain antibiotics in general wards and was usually conducted by a nurse without regard to allergic reaction history. However, the number of antibiotics tested varied from three to five. Among 12 hospitals, the ASTs for penicillins and cephalosporins were performed in 10 and 11 hospitals, respectively. In one hospital (E, Table 1), ASTs for cephalosporins were not routinely performed based on the idea that no study has demonstrated the clinical utility of cephalosporin skin testing for screening in subjects without a drug allergy history. Moreover, in two hospitals (H and I, Table 1), ASTs for penicillins were not performed because penicillins were never prescribed in these hospitals for fear of an adverse reaction. For other beta-lactam antibiotics, semisynthetic penicillins such as ampicillin and amoxicillin were tested in eight hospitals, carbapenems such as imipenem and meropenem in nine hospitals, and monobactams such as aztreonam in one hospital. Additionally, ASTs for vancomycin were conducted in four hospitals, and ASTs for aminoglycosides such as streptomycin were carried out in three hospitals. The details are shown in Table 1. Antibiotics other than those listed above were used without prior skin tests in all hospitals.

The ASTs were performed on the volar forearm using the intradermal method. However, skin tests using a control solution (e.g., saline) were not routinely conducted with the AST. Skin tests with saline were performed as a negative control in only two hospitals (F and G, Table 2), 
Table 2. Injection volume and interpretation of antibiotic skin testing in 12 Korean hospitals

\begin{tabular}{|c|c|c|c|}
\hline \multirow[t]{2}{*}{ Hospitals } & \multirow[t]{2}{*}{ Injection amount, mL } & \multicolumn{2}{|c|}{ Reading } \\
\hline & & After administration, min & Positive result \\
\hline$A$ & Make a bleb, 5 - $10 \mathrm{~mm}$ & $15-20$ & $W>10 \mathrm{~mm}$ or $E>15 \mathrm{~mm}$ \\
\hline B & $0.2-0.3$ & 15 & $W>5 \mathrm{~mm}$ \\
\hline C & 0.02 & 15 & W > initial \\
\hline $\mathrm{D}$ & 0.02 & $15-20$ & $W>10 \mathrm{~mm}$ or $E>15 \mathrm{~mm}$ \\
\hline$E$ & 0.02 & $15-20$ & $\mathrm{~W}>4 \mathrm{~mm}$ and $\mathrm{E}(+)$ \\
\hline $\mathrm{F}$ & 0.1 & $15-20$ & $\mathrm{E}>10 \mathrm{~mm}$ \\
\hline G & Make a bleb, $3 \mathrm{~mm}$ & $15-20$ & $W>1.5$ times \\
\hline $\mathrm{H}$ & 0.02 & $15-20$ & $W>$ initial or $E(+)$ \\
\hline 1 & Make a bleb, $3 \mathrm{~mm}$ & 15 & W > initial \\
\hline$J^{a}$ & 0.1 & $15-20$ & $W>10 \mathrm{~mm}$ or $\mathrm{E}>15 \mathrm{~mm}$ \\
\hline $\mathrm{K}^{\mathrm{a}}$ & 0.1 & 15 & W > initial or E $(+)$ \\
\hline$L^{a}$ & 0.1 & $15-20$ & $W>10 \mathrm{~mm}$ or $E>10 \mathrm{~mm}$ \\
\hline
\end{tabular}

W, wheal; E, erythema.

a Secondary hospitals; the others are tertiary hospitals.

following the AST and for suspicious, false-positive results cases. The injection amounts varied among the hospitals, ranging from 0.02 to $0.3 \mathrm{~mL}$ (Table 2). The concentrations tested also varied among hospitals (Table 1). For example, testing concentrations for penicillin $\mathrm{G}$ showed a 10,000 fold difference, ranging from $5 \mathrm{IU} / \mathrm{mL}$ to $50,000 \mathrm{IU} / \mathrm{mL}$. However, the time lag ( 15 to 20 minutes) between testing and interpretation was similar in all hospitals. The criteria for positive results on the AST varied and were inconsistent among the hospitals: either a wheal or erythema larger than standard in six hospitals, only a wheal regardless of erythema in four hospitals, and only erythema in one hospital. Only one hospital considered both a wheal and erythema for interpreting the results. Additionally, the wheal and erythema standards for a positive response varied among the hospitals (Table 2).

\section{DISCUSSION}

We examined the current practice of AST in 12 Korean general hospitals with different grades and locations. We emphasize that what we examined in this study was the routine testing performed for screening in all patients in general wards and not what allergy specialists do. Unexpectedly, the AST protocols varied from hospital to hospital with regard to the types of antibiotics tested, testing concentrations and amounts, and the interpretation method. Therefore, discussing the similarities and differences between the current practices and the commonly recommended procedures in the medical literature is warranted.

\section{AST methods}

Some experts recommend that the general method for the AST is an intradermal test after confirming a negative skin prick test [8-10]. Intradermal testing is performed on the volar surface of the forearm with a 0.02 to $0.05 \mathrm{~mL}$ injection of the reagent solution, raising a $3 \mathrm{~mm}$ wheal, with saline as the negative control and with histamine as the positive control [8-10]. Similarly, all hospitals enrolled in the present study conducted AST using intradermal methods. Because the AST protocol in this study was the practice performed in wards, only intradermal testing was performed. However, intradermal testing was performed without any control, which makes the interpretation of the test results more difficult and increases the likelihood of a false-negative or false-positive result. Moreover, the volumes injected varied from one to ten times more than the volume recommended in the medical literature. Although the intradermal test is thought to be relatively safe, it may induce severe reactions, such as anaphylaxis, especially when a large volume is injected. Considering this, AST should be performed using the proper volume of antibiotics.

The antibiotic concentrations tested should not cause any significant irritation in subjects with good tolerance to antibiotics. Currently, the maximum concentrations used 
Table 3. Beta-lactam concentrations recommended for skin testing

\begin{tabular}{|c|c|c|}
\hline Hapten & USA $^{a}$ & Europe $^{b}$ \\
\hline Penicillin G, IU/mL & 10,000 & 10,000 \\
\hline $\mathrm{PPL}, \mathrm{mmol} / \mathrm{L}$ & $6 \times 10^{-5}$ & $6 \times 10^{-6}$ \\
\hline MDM, mmol/L & $10^{-2}$ & $2 \times 10^{-2}$ \\
\hline Cephalosporin, mg/mL & $2-3$ & 2 \\
\hline Semisynthetic penicillin ${ }^{c}, \mathrm{mg} / \mathrm{mL}$ & - & 20 \\
\hline
\end{tabular}

PPL, penicilloyl-polylysine; MDM, minor determinant mixture.

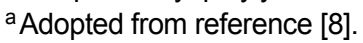

${ }^{\mathrm{b}}$ Adopted from references $[9,10]$.

${ }^{\mathrm{c}}$ Ampicillin, amoxicillin, and piperacillin.

differ from the accepted guidelines recommended by the American Academy of Allergy, Asthma and Immunology (AAAAI) / Joint Council of Allergy Asthma and Immunology (JCAAI) [8], the European Network for Drug Allergy (ENDA) / European Academy of Allergy, and the European Academy of Allergy and Clinical Immunology (EAACI) [9,10], as shown in Table 3. Notably, the testing concentrations in the hospitals we studied varied widely from one-thousandth less to ten times more than those recommended by published guidelines [8-10]. This is an important issue because testing with too low an antibiotic concentration can result in a false-negative, and too high a concentration can cause irritation and a false-positive result. Furthermore, high concentrations of antibiotics elevate the risk for anaphylaxis.

\section{AST interpretation}

Interpretation is recommended 15 to 20 minutes after the injection $[3,8-10]$. For the intradermal test, the wheal area should be marked initially and again 15 to 20 minutes later, and an increase in the diameter greater than $3 \mathrm{~mm}$ is considered positive [3,8-10]. As mentioned above, we found that the criteria for a positive result varied too much and were inconsistent among the hospitals, although the time lag between test and interpretation was similar (15 to 20 minutes). These inconsistencies made a comparison of test results among hospitals difficult.

\section{Selection of antibiotics tested}

AST is recommended as a routine practice for patients with suspected immediate-type allergy to penicillins (including semisynthetic penicillins) and cephalosporins but not to other antibiotics [11-16]. The proportion of patients labeled as allergic to penicillin who have a true IgE-medicated reaction to penicillin is very low $[8,17,18]$.
Among these patients, over $80 \%$ have negative skin test results and no allergic reaction if challenged, whereas most patients with positive skin test results will react if challenged [19]. Penicillin skin testing has a high negative predictive value and is reliable for identifying individuals at risk for an immediate allergic reaction to penicillins $[11,20,21]$. Recent studies have shown that using the penicillin skin test helps determine an appropriate antibiotic treatment for patients with a history of penicillin allergy and reduces the use of broad-spectrum antibiotics, which may be a useful strategy for managing antibiotic resistance $[11,19,22,23]$. Many patients with a history of penicillin allergy and positive penicillin skin test results have a vague history of a prior reaction to penicillin [24]. In other words, the patient history may not be accurate, and penicillin skin testing should be considered for confirmation.

The increased use of beta-lactam antibiotics other then penicillin, such as amoxicillin, ampicillin, and cephalosporins, has increased the chance for allergic reactions to these antibiotics; thus, evaluating primary sensitization and cross-reactivities is important. The AST value for semisynthetic penicillins has been assessed extensively [10,25-27]. Semisynthetic penicillins may induce specific reactions and skin test positivity without cross-reacting to penicillins, which makes it necessary to include semisynthetic penicillins in ASTs. However, the negative predictive value of skin testing is unknown for cephalosporins $[15,28]$. Previous studies have reported a high negative predictive value when using parent cephalosporins at $2 \mathrm{mg} / \mathrm{mL}$, but further study is needed for confirmation $[16,29]$. Moreover, cross-reactivity information between penicillins and cephalosporins is lacking. Considering a $4.4 \%$ rate of cross-reaction to cephalosporins in patients with a history of penicillin allergy and a positive result on a penicillin skin test [30], cephalosporin skin testing should be performed in patients with a history of penicillin allergy. The AST is not only a relatively simple and safe procedure but also a minimally required procedure to avoid medical / legal problems.

The usefulness of skin testing for carbapenems has not been determined. Moreover, the cross-reactivity between penicillins and carbapenems is unclear, as studies have reported conflicting findings [21,31-35]. Recently, Romano et al. [35] published two prospective studies on the utility of skin testing to detect immediate-type allergic reactions to imipenem-cilastatin and meropenem [36]. Because skin testing with an unmetabolized drug is 
unlikely to detect all sensitive individuals, patients with a negative reaction underwent a graded challenge, and no patients reacted. The authors concluded that this approach appeared safe, that the cross-reactivity was better defined, and that carbapenem reagents for skin testing are available. However, further confirmation is needed.

Many hospitals enrolled in the present study performed ASTs with antibiotics such as vancomycin, streptomycin, and monobactam that have not yet been validated. Up to five different antibiotics were identified in six hospitals (A, B, C, E, J, and K, Table 1). An AST should be performed to identify immediate-type allergic reactions to penicillins and cephalosporins, as discussed above, and not to other antibiotics in which the reliability of AST has not been validated.

This inconsistency in the AST procedure can result in a waste of time, money, labor, and the nation's resources and can put patients at risk. Moreover, inconsistencies in the AST procedure make it difficult to conduct comparative studies on adverse antibiotic events among countries and hospitals. Of course, we should not accept other countries guidelines blindly but make our guidelines adequate for our circumstances. In conclusion, the practice of AST performed in hospital wards in Korea was too varied and inconsistent. The standardized guidelines for AST must be followed for the safe and effective use of antibiotics in Korea.

\section{Conflict of interest}

No potential conflict of interest relevant to this article was reported.

\section{Acknowledgements}

We thank to Dr. Soo-Gul Lee, professor at Dong-A University Medical Center; Dr. Hae-Kyung Park, professor at Busan National University Hospital; Dr. Byung-Jae Lee, professor at Samsung Medical Center; Dr. Tae-Bum Kim, professor at the Asan Medical Center; Dr. Gyu-Young Hur, professor at the Korea University College of Medicine; Dr. Yong-Won Lee of the Yonsei University College of Medicine; Dr. In-Seon Choi, professor at Chonnam National University Hospital; Dr. Yong-Eun Kwon, professor at Chosun University Hospital; Dr. SookHee Song of the Seoul Medical Center; Dr. Hee-Kyoo Kim, professor at Kosin University College of Medicine; and Dr. Sang-Rok Lee of Cheongju St. Mary's Hospital. This study was supported by a grant from the Korea Health 21 R\&D
Project, Ministry for Health, Welfare and Family Affairs, Korea (A030001).

\section{REFERENCES}

1. Lee CE, Zembower TR, Fotis MA, et al. The incidence of antimicrobial allergies in hospitalized patients: implications regarding prescribing patterns and emerging bacterial resistance. Arch Intern Med 2000;160:2819-2822.

2. Arroliga ME, Wagner W, Bobek MB, et al. A pilot study of penicillin skin testing in patients with a history of penicillin allergy admitted to a medical ICU. Chest 2000;118:1106-1108.

3. Torres MJ, Blanca M, Fernandez J, et al. Diagnosis of immediate allergic reactions to beta-lactam antibiotics. Allergy 2003;58:961972.

4. Fridkin SK, Edwards JR, Courval JM, et al. The effect of vancomycin and third-generation cephalosporins on prevalence of vancomycin-resistant enterococci in 126 U.S. adult intensive care units. Ann Intern Med 2001;135:175-183.

5. Salgado CD, Farr BM. Outcomes associated with vancomycinresistant enterococci: a meta-analysis. Infect Control Hosp Epidemiol 2003;24:690-698.

6. Loo VG, Poirier L, Miller MA, et al. A predominantly clonal multiinstitutional outbreak of Clostridium difficile-associated diarrhea with high morbidity and mortality. N Engl J Med 2005;353:24422449.

7. Neuhauser MM, Weinstein RA, Rydman R, et al. Antibiotic resistance among gram-negative bacilli in US intensive care units: implications for fluoroquinolone use. JAMA 2003;289:885-888.

8. Executive summary of disease management of drug hypersensitivity: a practice parameter. Joint Task Force on Practice Parameters, the American Academy of Allergy, Asthma and Immunology, and the Joint Council of Allergy, Asthma and Immunology. Ann Allergy Asthma Immunol 1999;83:665-700.

9. Brockow K, Romano A, Blanca M, et al. General considerations for skin test procedures in the diagnosis of drug hypersensitivity. Allergy 2002;57:45-51.

10. Torres MJ, Romano A, Mayorga C, et al. Diagnostic evaluation of a large group of patients with immediate allergy to penicillins: the role of skin testing. Allergy 2001;56:850-856.

11. del Real GA, Rose ME, Ramirez-Atamoros MT, et al. Penicillin skin testing in patients with a history of beta-lactam allergy. Ann Allergy Asthma Immunol 2007;98:355-359.

12. Park MA, Li JT. Diagnosis and management of penicillin allergy. Mayo Clin Proc 2005; 80:405-410.

13. Gruchalla RS, Pirmohamed M. Clinical practice. Antibiotic allergy. N Engl J Med 2006; 354:601-609.

14. Blanca M, Romano A, Torres MJ, Demoly P, DeWeck A. Continued need of appropriate betalactam-derived skin test reagents for the management of allergy to betalactams. Clin Exp Allergy 2007; 37:166-173. 
15. Greenberger PA. 8. Drug allergy. J Allergy Clin Immunol 2006; 117:S464-S470.

16. Romano A, Guéant-Rodriguez RM, Viola M, Pettinato R, Gueant JL. Cross-reactivity and tolerability of cephalosporins in patients with immediate hypersensitivity to penicillins. Ann Intern Med 2004;141:16-22.

17. Sogn DD, Evans R 3rd, Shepherd GM, et al. Results of the national institute of allergy and infectious diseases collaborative clinical trial to test the predictive value of skin testing with major and minor penicillin derivatives in hospitalized adults. Arch Intern Med 1992;152:1025-1032.

18. Arroliga ME, Wagner W, Bobek MB, et al. A pilot study of penicillin skin testing in patients with a history of penicillin allergy admitted to a medical ICU. Chest 2000;118:1106-1108.

19. Nadarajah K, Green GR, Naglak M. Clinical outcomes of penicillin skin testing. Ann Allergy Asthma Immunol 2005;95:541-545.

20. Salkind AR, Cuddy PG, Foxworth JW. The rational clinical examination. Is this patient allergic to penicillin? An evidencebased analysis of the likelihood of penicillin allergy. JAMA 2001;285:2498-2505.

21. Weiss ME, Adkinson NF. Immediate hypersensitivity reactions to penicillin and related antibiotics. Clin Allergy 1988;18:515-540.

22. Arroliga ME, Radojicic C, Gordon SM, et al. A prospective observational study of the effect of penicillin skin testing on antibiotic use in the intensive care unit. Infect Control Hosp Epidemiol 2003;24:347-350.

23. Yates R. New intervention strategies for reducing antibiotic resistance. Chest 1999;115:24S-27S.

24. Solensky R, Earl HS, Gruchalla RS. Penicillin allergy: prevalence of vague history in skin test-positive patients. Ann Allergy Asthma Immunol 2000;85:195-199.

25. Torres MJ, Mayorga C, Pamies R, et al. Immunologic response to different determinants of benzylpenicillin, amoxicillin, and ampicillin. Comparison between urticaria and anaphylactic shock. Allergy 1999;54:936-943.
26. Silviu-Dan F, McPhillips S, Warrington RJ. The frequency of skin test reactions to side-chain penicillin determinants. J Allergy Clin Immunol 1993;91:694-701.

27. Bousquet PJ, Co-Minh HB, Arnoux B, Daures JP, Demoly P. Importance of mixture of minor determinants and benzylpenicilloyl poly-L-lysine skin testing in the diagnosis of beta-lactam allergy. $\mathrm{J}$ Allergy Clin Immunol 2005;115:1314-1316.

28. Madaan A, Li JT. Cephalosporin allergy. Immunol Allergy Clin North Am 2004;24:463-476.

29. Romano A, Guéant-Rodriguez RM, Viola M, et al. Diagnosing immediate reactions to cephalosporins. Clin Exp Allergy 2005; 35:1234-1242.

30. Kelkar PS, Li JT. Cephalosporin allergy. N Engl J Med 2001; 345:804-809.

31. Sodhi M, Axtell SS, Callahan J, Shekar R. Is it safe to use carbapenems in patients with a history of allergy to penicillin? $\mathrm{J}$ Antimicrob Chemother 2004;54:1155-1157.

32. Prescott WA Jr, DePestel DD, Ellis JJ, Regal RE. Incidence of carbapenem-associated allergic-type reactions among patients with versus patients without a reported penicillin allergy. Clin Infect Dis 2004;38:1102-1107.

33. Saxon A, Adelman DC, Patel A, Hajdu R, Calandra GB. Imipenem cross-reactivity with penicillin in humans. J Allergy Clin Immunol 1988;82:213-217.

34. McConnell SA, Penzak SR, Warmack TS, Anaissie EJ, Gubbins PO. Incidence of imipenem hypersensitivity reactions in febrile neutropenic bone marrow transplant patients with a history of penicillin allergy. Clin Infect Dis 2000;31:1512-1514.

35. Romano A, Viola M, Guéant-Rodriguez RM, et al. Imipenem in patients with immediate hypersensitivity to penicillins. N Engl J Med 2006;354:2835-2837.

36. Romano A, Viola M, Guéant-Rodriguez RM, et al. Brief communication: tolerability of meropenem in patients with IgEmediated hypersensitivity to penicillins. Ann Intern Med 2007;146:266-269. 